\title{
RESEARCH ARTICLE \\ Measures of Extropy for Concomitants of Generalized Order Statistics in Morgenstern Family
}

\author{
Zahra Almaspoor $^{1} \cdot$ Ali Akbar Jafari ${ }^{1}$ (D) Saeid Tahmasebi ${ }^{2}$
}

Received: 25 August 2021 / Accepted: 16 December 2021 / Published online: 3 January 2022

(c) The Author(s) 2021

\begin{abstract}
In this paper, a measure of extropy is obtained for concomitants of m-generalized order statistics in the Morgenstern family. The cumulative residual extropy (CREX) and negative cumulative extropy (NCEX) are presented for the $r$ th concomitant of m-generalized order statistics. In addition, the problem of estimating the CREX and NCEX is studied utilizing the empirical method in concomitants of m-generalized order statistics. Some applications of these results are given for the concomitants of order statistics and record values.
\end{abstract}

Keywords Concomitants · Cumulative residual extropy · Extropy measure · Generalized order statistics $\cdot$ Negative cumulative extropy

MSC Classification 62B10 $\cdot 62 \mathrm{G} 30$

$\begin{array}{ll}\text { Abbreviations } \\ \text { cdf } & \text { Cumulative distribution function } \\ \text { pdf } & \text { Probability density function } \\ \text { GOSs } & \text { Generalized order statistics } \\ \text { CREX } & \text { Cumulative residual extropy } \\ \text { NCEX } & \text { Negative cumulative extropy } \\ \text { NCREX } & \text { Negative cumulative residual extropy } \\ \text { MTEWD } & \text { Morgenstern type bivariate extended Weibull distribution } \\ \text { MTED } & \text { Morgenstern type bivariate exponential distribution } \\ \text { MTUD } & \text { Morgenstern type bivariate uniform distribution }\end{array}$

Ali Akbar Jafari

aajafari@yazdu.ac.ir

Zahra Almaspoor

zalmaspoor@yahoo.com

Saeid Tahmasebi

tahmasebi@pgu.ac.ir

1 Department of Statistics, Yazd University, Yazd, Iran

2 Department of Statistics, Faculty of Intelligent Systems Engineering and Data Science, Persian Gulf University, Bushehr, Iran 


\section{Introduction}

The concept of generalized order statistics (GOSs) has been introduced by [1] as a unified model for ascendingly ordered random variables. It is included a variety of models of ordered random variables such as ordinary order statistics, sequential order statistics, progressive type-II censoring, record values, and Pfeifer's records. Let $F$ be an absolutely continuous cumulative distribution function (cdf) with corresponding probability density function (pdf) $f$. The random variables $\boldsymbol{X}=\left(X_{(1, n, \tilde{m}, k)}, \ldots, X_{(n, n, \tilde{m}, k)}\right)$ are GOSs based on $F$ if their joint pdf is

$$
f_{\boldsymbol{X}}\left(x_{1}, \ldots, x_{n}\right)=C_{n}\left(\prod_{i=1}^{n-1}\left(1-F\left(x_{i}\right)\right)^{\gamma_{i}-\gamma_{i+1}-1} f\left(x_{i}\right)\right)\left(1-F\left(x_{n}\right)\right)^{k-1} f\left(x_{n}\right),
$$

where $\quad F^{-1}(0)<x_{1}<\cdots<x_{n}<F^{-1}(1), \quad C_{r}=\prod_{i=1}^{r} \gamma_{i}, \quad r=1, \ldots n, \quad$ and $F^{-1}(t)=\inf \{x: F(x) \geq t\}$ denotes the quantile function of $F$. The parameters in (1) are defined by $\gamma_{i}=k+n-i+\sum_{l=i}^{n-1} m_{l}>0, i=1, \ldots, n-1, \gamma_{n+1}=0, \gamma_{n}=k$, and $\tilde{m}=\left(m_{1}, \ldots, m_{n-1}\right) \in \mathbb{R}^{n-1}$.

Appropriate choices of the parameters $m_{i}$ and $k$ lead to special cases of GOSs. For example, the GOSs $X_{(r, n, \tilde{m}, k)}$ reduces to the $r$ th order statistic choosing $m_{i}=0$ and $k=1$, whereas it becomes the $r$ th upper record for $m_{i}=-1$ and $k=1$ ([1]). The GOSs also include sequential order statistics, $k$-record values, Pfeifer's records, and progressive type-II censored statistics. In this paper, we consider $m$-GOSs and denote the random variable $X_{(r, n, \tilde{m}, k)}$ by $X_{(r, n, m, k)}, r=1, \ldots, n$. The marginal pdf of $X_{(r, n, m, k)}$ is

$$
f_{(r, n, m, k)}(x)=\frac{C_{r}}{(r-1) !} \bar{F}^{\gamma_{r}-1}(x) f(x) h_{m}^{r-1}(F(x)),
$$

where $\bar{F}=1-F$ and

$$
h_{m}(F(x))= \begin{cases}\frac{1}{m+1}\left[1-\bar{F}^{m+1}(x)\right] & m \neq-1 \\ -\log \bar{F}(x) & m=-1, F(x) \in[0,1) .\end{cases}
$$

Suppose that $\left(X_{i}, Y_{i}\right), i=1,2, \ldots$ is a sequence of independent random variables from a bivariate distribution. If the $X$-variates are arranged in increasing order as $X_{(1, n, m, k)} \leq X_{(2, n, m, k)} \leq \cdots \leq X_{(n, n, m, k)}$, then $Y$-variates paired with these $m$-GOSs are called the concomitants of $m$-GOSs and denoted by $Y_{[r, n, m, k]}, r=1, \ldots, n$. For the Farlie-Gumbel-Morgenstern (FGM) family defined by [2] with pdf given by

$$
f_{X, Y}(x, y)=f_{X}(x) f_{Y}(y)\left[1+\alpha\left(2 F_{X}(x)-1\right)\left(2 F_{Y}(y)-1\right)\right], \quad|\alpha| \leq 1,
$$

the pdf and cdf of the concomitant of the $r$ th $m$-GOS are given by [3] as follows:

$$
\begin{aligned}
& g_{[r, n, m, k]}(y)=f_{Y}(y)\left[1+\alpha C^{*}(r, n, m, k)\left(1-2 F_{Y}(y)\right)\right], \\
& G_{[r, n, m, k]}(y)=F_{Y}(y)\left[1+\alpha C^{*}(r, n, m, k)\left(1-F_{Y}(y)\right)\right],
\end{aligned}
$$

where $C^{*}(r, n, m, k)=\frac{2 \prod_{j=1}^{r} \gamma_{j}}{\prod_{i=1}^{r}\left(\gamma_{i}+1\right)}-1$ 
The concomitants of $m$-GOSs in the FGM family were studied by [3]. Some properties for the concomitants of $m$-GOSs from FGM type bivariate Rayleigh distribution were obtained by [4]. Some inaccuracy measures for the concomitants of $m$-GOSs in the FGM family were derived by [5. Barakat and Husseiny [6] and Abd Elgawad et al.7] studied some information measures in concomitants of $m$-GOSs under iterated FGM and Huang-Kotz FGM, respectively. Abd Elgawad et al. [8] and Alawady et al. [9] investigated some properties of concomitants of $m$-GOS from the bivariate Cambanis family. Mohamed et al. [10] studied the residual extropy of concomitants of $m$-GOSs based on FGM distribution and presented a nonparametric estimation for this measure.

Recently, an alternative measure of uncertainty, named by extropy, was proposed by [11]. For an absolutely continuous nonnegative random variable $X$ with pdf $f$ and cdf $F$, the extropy is defined as

$$
J(X)=-\frac{1}{2} \int_{0}^{+\infty}[f(x)]^{2} \mathrm{~d} x=-\frac{1}{2} \int_{0}^{1} f\left(F^{-1}(u)\right) d u .
$$

Some authors paid attention to extropy and its applications. Qui [12] discussed some characterization results, and lower bounds of extropy for order statistics and record values. Qiu and Jia [13] studied the residual extropy of order statistics and [14] explored the extropy estimators with applications in testing uniformity. Qiu et al. [15] obtained some results on the extropy properties of mixed systems. Zamanzade and Mahdizadeh [16] presented an extropy-based test of uniformity in ranked set sampling and compared it with simple random sampling. Mohamed et al.[17] applied the fractional and weighted cumulative residual entropy measures to test the uniformity and discussed some of its properties.

The cumulative residual extropy (CREX) was proposed by [18] in analogy with (5) as a measure of uncertainty of random variables. The CREX is defined as

$$
\mathcal{J}^{\star}(X)=-\frac{1}{2} \int_{0}^{\infty} \bar{F}^{2}(x) \mathrm{d} x .
$$

It is always non-positive. Hence, the negative CREX (NCREX) can be presented as

$$
\mathcal{J}(X)=\frac{1}{2} \int_{0}^{\infty} \bar{F}^{2}(x) \mathrm{d} x .
$$

[18] and [19] studied and investigated some results on the CREX and hence NCREX. Recently, [20] proposed a negative cumulative extropy (NCEX) in analogy with (7), defined as

$$
\mathcal{C} \mathcal{J}(X)=\frac{1}{2} \int_{0}^{\infty}\left[1-F^{2}(x)\right] \mathrm{d} x=\frac{1}{2} \int_{0}^{1} \frac{\phi(u)}{f\left(F^{-1}(u)\right)} d u,
$$

where $\phi(u)=\frac{1-u^{2}}{2}, 0<u<1$.

Motivated by $[5,10,16,18,20]$, in this paper, we aim to present some results on extropy for concomitants of $m$-GOSs in the FGM family. Therefore, the rest of 
this paper is organized as follows: In Sect. 2, we first obtain a measure of extropy for $Y_{[r, n, m, k]}$ in the FGM family. We also study some results of CREX and NCEX for $G_{[r, n, m, k]}(y)$. In Sect. 3, we discuss the problem of estimating the NCREX and NCEX employing the empirical NCREX and NCEX for concomitants of $m$-GOSs. A real example is given in Sect. 4.

Note that the terms increasing and decreasing are used in non-strict sense. Throughout this paper, it is assumed that the expectation exists when it appears.

\section{Some Measures for $Y_{[r, n, m, k]}$}

In this section, we obtain the measures of extropy, CREX, and NCEX for the $r$ th concomitant $Y_{[r, n, m, k]}$ of $m$-GOSs in FGM family. Some properties of these measures are investigated, and applications of this result are given for the concomitants of order statistics and record values.

\subsection{Extropy Measure for $Y_{[r, n, m, k]}$}

If $Y_{[r, n, m, k]}$ is the concomitant of the $r$ th $m$-GOSs with pdf (3), then the extropy measure is given by

$$
\begin{aligned}
J\left(Y_{[r, n, m, k]}\right)= & -\frac{1}{2} \int_{0}^{\infty} g_{[r, n, m, k]}^{2}(y) d y \\
= & J(Y) \\
& -\frac{\left[\alpha C^{*}(r, n, m, k)\right]^{2}}{2} \mathbb{E}\left[f_{Y}\left(F_{Y}^{-1}(U)\right)(1-2 U)^{2}\right] \\
& -\alpha C^{*}(r, n, m, k) \mathbb{E}\left[f_{Y}\left(F_{Y}^{-1}(U)\right)(1-2 U)\right], \\
= & J(Y)\left[1+\alpha C^{*}(r, n, m, k)\right]^{2} \\
& +2 \alpha C^{*}(r, n, m, k) \mathbb{E}\left[f_{Y}\left(F_{Y}^{-1}(U)\right) U\right] \\
& -2\left[\alpha C^{*}(r, n, m, k)\right]^{2} \mathbb{E}\left[f_{Y}\left(F_{Y}^{-1}(U)\right)\left(U^{2}-U\right)\right],
\end{aligned}
$$

where $U$ is a uniformly random variable on $(0,1)$, and $J(Y)$ is the extropy of the random variable $Y$.

Suppose that $Q(u)=F_{Y}^{-1}(u)$ is the quantile function. We know that $q(u) f_{Y}(Q(u))=1$, where $q(u)$ is derivative of $Q(u)$ with respect to $u$, i.e. $q(u)=Q^{\prime}(u)$ that it is known as the quantile density function. Now by making use of (9), the corresponding quantile-based $J\left(Y_{[r, n, m, k]}\right)$ can be written as

$$
\begin{aligned}
J\left(Y_{[r, n, m, k]}\right)= & J(Y)-\frac{\left[\alpha C^{*}(r, n, m, k)\right]^{2}}{2} \mathbb{E}\left[\frac{(1-2 U)^{2}}{q(U)}\right] \\
& -\alpha C^{*}(r, n, m, k) \mathbb{E}\left[\frac{1-2 U}{q(U)}\right]
\end{aligned}
$$


where $J(Y)=\frac{-1}{2} \mathbb{E}\left[\frac{1}{q(U)}\right]$. This quantity is easy to obtain. In the following, we consider order statistics and record values as two special cases of $m$-GOSs and obtain the properties of extropy measure for their concomitant.

Case 1: If $m=0$ and $k=1$, then the $m$-GOSs become order statistics. The pdf and cdf of the concomitant of the $r$ th order statistic, $Y_{[r: n]}$, are given by

$$
\begin{aligned}
& g_{Y_{[r: n]}}(y)=f_{Y}(y)\left[1+\alpha \delta_{r}\left(1-2 F_{Y}(y)\right)\right], \\
& G_{Y_{[r: n]}}(y)=F_{Y}(y)\left[1+\alpha \delta_{r}\left(1-F_{Y}(y)\right)\right],
\end{aligned}
$$

respectively, where $\delta_{r}=\frac{n-2 r+1}{n+1}$. According to (10), the extropy measure of $Y_{[r: n]}$ is obtained as

$$
J\left(Y_{[r: n]}\right)=J(Y)-\frac{\left(\alpha \delta_{r}\right)^{2}}{2} \mathbb{E}\left[\frac{(1-2 U)^{2}}{q(U)}\right]-\alpha \delta_{r} \mathbb{E}\left[\frac{1-2 U}{q(U)}\right] .
$$

Define now $a_{n}=\frac{n-1}{n+1}$. From (11), we have

$$
\begin{aligned}
& J\left(Y_{[n: n]}\right)=J(Y)-\frac{\left(\alpha a_{n}\right)^{2}}{2} \mathbb{E}\left[\frac{(1-2 U)^{2}}{q(U)}\right]+\alpha a_{n} \mathbb{E}\left[\frac{1-2 U}{q(U)}\right], \\
& J\left(Y_{[1: n]}\right)=J(Y)-\frac{\left(\alpha a_{n}\right)^{2}}{2} \mathbb{E}\left[\frac{(1-2 U)^{2}}{q(U)}\right]-\alpha a_{n} \mathbb{E}\left[\frac{1-2 U}{q(U)}\right] .
\end{aligned}
$$

Therefore,

$$
J\left(Y_{[n: n]}\right)-J\left(Y_{[1: n]}\right)=2 \alpha a_{n} \mathbb{E}\left[\frac{1-2 U}{q(U)}\right]=-4 \alpha a_{n}\left(J(Y)+\mathbb{E}\left[\frac{U}{q(U)}\right]\right) .
$$

Moreover, if $\lambda \geq 1$ is an integer number and we change $r$ to $r \lambda$ and $n$ to $(n+1) \lambda-1$, then, from (11), we have

$$
J\left(Y_{[r: n]}\right)=J\left(Y_{[r \lambda:(n+1) \lambda-1]}\right) .
$$

In the following, we present some examples and properties of $J\left(Y_{[r: n]}\right)$.

Example 1 Suppose that we have the Morgenstern type bivariate extended Weibull distribution (MTEWD) with cdf

$$
\begin{array}{r}
F(x, y)=\left[1-e^{-\theta_{1} H\left(x ; \xi_{1}\right)}\right]\left[1-e^{-\theta_{2} H\left(y ; \xi_{2}\right)}\right]\left[1+\alpha e^{-\theta_{1} H\left(x ; \xi_{1}\right)-\theta_{2} H\left(y ; \xi_{2}\right)}\right], \\
x>0, \quad y>0,
\end{array}
$$

where $\theta_{i}>0, \boldsymbol{\xi}_{i}$ is a vector of parameters, and $H(x ; \boldsymbol{\xi})$ is a non-negative, continuous, monotone increasing, and differentiable function of $x$ which depends on the parameter vector $\boldsymbol{\xi}$. Also, $H(x ; \xi) \rightarrow 0^{+}$as $x \rightarrow 0^{+}$and $H(x ; \xi) \rightarrow \infty$ as $x \rightarrow \infty$. From (11), we can find 


$$
\begin{aligned}
J\left(Y_{[r: n]}\right) & =-\frac{1}{2} \int_{0}^{\infty} g_{[r: n]}^{2}(y) d y \\
& =-\frac{1}{2} \int_{0}^{\infty}\left[\theta_{2} h\left(y ; \xi_{2}\right) e^{-\left(\theta_{2} H\left(y ; \xi_{2}\right)\right)}\left(1+\alpha \delta_{r}\left(2 e^{-\theta_{2} H\left(y ; \xi_{2}\right)}-1\right)\right)\right]^{2} d y \\
& =-\frac{1}{2} \int_{0}^{\infty}\left[f_{Y}(y)\left(1-\alpha \delta_{r}\right)+\alpha \delta_{r} f_{W}(y)\right]^{2} d y \\
& =J(Y)\left[1-\alpha \delta_{r}\right]^{2}+\left(\alpha \delta_{r}\right)^{2} J(W)+\frac{16}{9}\left[\alpha \delta_{r}-\left(\alpha \delta_{r}\right)^{2}\right] J(V),
\end{aligned}
$$

where $W$ and $V$ follow $E W\left(2 \theta_{2}, \xi_{2}\right)$ and $E W\left(\frac{3 \theta_{2}}{2}, \xi_{2}\right)$, respectively, and $E W(\theta, \xi)$ denotes the extended Weibull distribution with the following cdf:

$$
F(x)=1-e^{-\theta H(x ; \xi)}, x>0 .
$$

In the following, we consider some special cases of MTEWD.

Example 2 For the Morgenstern type bivariate exponential distribution (MTED) with cdf

$$
F(x, y)=\left(1-e^{-\frac{x}{\theta_{1}}}\right)\left(1-e^{-\frac{y}{\theta_{2}}}\right)\left[1+\alpha e^{-\frac{x}{\theta_{1}}-\frac{y}{\theta_{2}}}\right], x>0, \quad y>0,
$$

using (13), we have

$$
J\left(Y_{[r: n]}\right)=\frac{1}{12 \theta_{2}}\left[3-\left(\alpha \delta_{r}\right)^{2}-2 \alpha \delta_{r}\right] .
$$

Also, we get

$$
A_{\alpha}(n)=J\left(Y_{[n: n]}\right)-J\left(Y_{[1: n]}\right)=\frac{\alpha}{3 \theta_{2}} a_{n}
$$

which is positive, negative or zero whenever $0<\alpha \leq 1,-1 \leq \alpha<0$, or $\alpha=0$, respectively. Also, the difference between $J\left(Y_{[r: n]}\right)$ and $J(Y)$ is

$$
B_{\alpha, n}(r)=J\left(Y_{[r: n]}\right)-J(Y)=-\frac{\alpha \delta_{r}}{12 \theta_{2}}\left(\alpha \delta_{r}+2\right) .
$$

$B_{\alpha, n}(r)$ is positive for $-1 \leq \alpha<0,1 \leq r<\frac{n+1}{2}$ (or $0<\alpha \leq 1, \frac{n+1}{n^{2}+1}<r \leq n$ ). Also, it is negative for $-1 \leq \alpha<0, \frac{n+1}{2}<r \leq n$ (or $0<\alpha \leq 1,1 \leq r<\frac{n^{2}+1}{2}$ ).

Example 3 For the Morgenstern type bivariate logistic distribution with the cdf

$$
\begin{aligned}
F(x, y)=(1+\exp (-x))^{-1}(1+\exp (-y))^{-1}( & \left.1+\frac{\alpha e^{-x-y}}{\left(1+e^{-x}\right)\left(1+e^{-y}\right)}\right), \\
& -\infty<x<+\infty,-\infty<y<+\infty
\end{aligned}
$$

using (13), we have 


$$
J\left(Y_{[r: n]}\right)=\frac{-1}{12}-\frac{\left(\alpha \delta_{r}\right)^{2}}{60}-\frac{\alpha \delta_{r}}{16}
$$

Also, we get

$$
D_{\alpha}(n)=J\left(Y_{[n: n]}\right)-J\left(Y_{[1: n]}\right)=\frac{\alpha}{8} a_{n},
$$

which is positive, negative or zero whenever $0<\alpha \leq 1,-1 \leq \alpha<0$ or $\alpha=0$, respectively.

Example 4 For the Morgenstern type bivariate Rayleigh distribution with the cdf

$$
F(x, y)=\left(1-\exp \left(-\frac{x^{2}}{2 \sigma_{1}^{2}}\right)\right)\left(1-\exp \left(-\frac{y^{2}}{2 \sigma_{2}^{2}}\right)\right)\left(1+\alpha \exp \left(-\frac{x^{2}}{2 \sigma_{1}^{2}}-\frac{y^{2}}{2 \sigma_{2}^{2}}\right)\right),
$$

we have

$$
J\left(Y_{[r: n]}\right)=-\frac{\sqrt{\pi}}{8 \sigma_{2}}-\frac{0.026\left(\alpha \delta_{r}\right)^{2}}{\sigma_{2}}-\frac{0.02\left(\alpha \delta_{r}\right)}{\sigma_{2}}
$$

Also,

$$
W_{\alpha}(n)=J\left(Y_{[n: n]}\right)-J\left(Y_{[1: n]}\right)=\frac{0.04 \alpha}{\sigma_{2}} a_{n},
$$

which is positive, negative or zero whenever $0<\alpha \leq 1,-1 \leq \alpha<0$ or $\alpha=0$, respectively.

Example 5 For the Morgenstern type bivariate exponentiated exponential distribution with the cdf

$$
F_{X, Y}(x, y)=\left[\left(1-e^{-\theta_{1} x}\right)\left(1-e^{-\theta_{2} y}\right)\right]^{\lambda}\left[1+\alpha\left(1-\left(1-e^{-\theta_{1} x}\right)^{\lambda}\right)\left(1-\left(1-e^{-\theta_{2} y}\right)^{\lambda}\right)\right],
$$

if $\lambda \geq 1$, we get

$$
\begin{aligned}
J\left(Y_{[r: n]}\right)= & -\frac{\lambda \theta_{2}}{4(2 \lambda-1)}-\frac{\left(\alpha \delta_{r}\right)^{2}}{2} \lambda \theta_{2}\left[\frac{\left(26 \lambda^{2}-9 \lambda+1\right)}{12(2 \lambda-1)(4 \lambda-1)(3 \lambda-1)}\right] \\
& -\alpha \delta_{r}\left[\frac{5 \lambda-1}{6(2 \lambda-1)(3 \lambda-1)}\right] .
\end{aligned}
$$

Finally, we have

$$
Q_{\alpha, \lambda}(n)=J\left(Y_{[n: n]}\right)-J\left(Y_{[1: n]}\right)=\alpha a_{n}\left[\frac{5 \lambda-1}{2(2 \lambda-1)(3 \lambda-1)}\right],
$$

which is positive, negative or zero whenever $0<\alpha \leq 1,-1 \leq \alpha<0$ or $\alpha=0$, respectively. 
Hereafter, we consider the concomitants of order statistics while $\left(X_{1}, Y_{1}\right)$, $\left(X_{2}, Y_{2}\right), \ldots,\left(X_{n}, Y_{n}\right)$ are independent but otherwise arbitrarily distributed. Let us consider the Morgenstern family with cdf

$$
F_{X, Y}^{i}(x, y)=F_{X}^{i}(x) F_{Y}^{i}(y)\left[1+\alpha_{i}\left(1-F_{X}^{i}(x)\right)\left(1-F_{Y}^{i}(y)\right)\right] .
$$

Now, suppose that $F_{X}^{i}(x)=F_{X}(x), F_{Y}^{i}(y)=F_{Y}(y)$ and $\left|\alpha_{i}\right| \leq 1$. Then, the pdf's of $Y_{[1: n]}$ and $Y_{[n: n]}$ are given by [21] as follows:

$$
\begin{aligned}
& g_{Y_{[1: n]}}(y)=f_{Y}(y)\left(1+b_{n} \sum_{j=1}^{n} \alpha_{j}\left(1-2 F_{Y}(y)\right)\right), \\
& g_{Y_{[n: n]}}(y)=f_{Y}(y)\left(1-b_{n} \sum_{j=1}^{n} \alpha_{j}\left(1-2 F_{Y}(y)\right)\right),
\end{aligned}
$$

where $b_{n}=\frac{n-1}{(n+1) n}$. Furthermore, the extropy measures for concomitants of extremes of order statistics are presented as

$$
\begin{aligned}
& J\left(Y_{[1: n]}\right)=J(Y)-\frac{\left[b_{n} \sum_{j=1}^{n} \alpha_{j}\right]^{2}}{2} \mathbb{E}\left[\frac{(1-2 U)^{2}}{q(U)}\right]-b_{n} \sum_{j=1}^{n} \alpha_{j} \mathbb{E}\left[\frac{1-2 U}{q(U)}\right], \\
& J\left(Y_{[n: n]}\right)=J(Y)-\frac{\left[b_{n} \sum_{j=1}^{n} \alpha_{j}\right]^{2}}{2} \mathbb{E}\left[\frac{(1-2 U)^{2}}{q(U)}\right]+b_{n} \sum_{j=1}^{n} \alpha_{j} \mathbb{E}\left[\frac{1-2 U}{q(U)}\right] .
\end{aligned}
$$

Hence, we have

$$
A_{n}=J\left(Y_{[n: n]}\right)-J\left(Y_{[1: n]}\right)=2 b_{n} \sum_{j=1}^{n} \alpha_{j} \mathbb{E}\left[\frac{1-2 U}{q(U)}\right] .
$$

Finally, we get

$$
J(Y)=\frac{1}{2}\left\{J\left(Y_{[n: n]}\right)+J\left(Y_{[1: n]}\right)+\left[b_{n} \sum_{j=1}^{n} \alpha_{j}\right]^{2} \mathbb{E}\left[\frac{(1-2 U)^{2}}{q(U)}\right]\right\}
$$

Case 2: Let $\left(X_{1}, Y_{1}\right),\left(X_{2}, Y_{2}\right), \cdots$ be a sequence of bivariate random variables from a continuous distribution. If $\left\{R_{r}, r \geq 1\right\}$ is the sequence of upper record values in the sequence of $X$ 's, then the $Y$ which corresponds with the $r$ th record will be called the concomitant of the $r$ th record, denoted by $R_{[r]}$. The concomitants of record values apply in practical experiments such as life time experiments, sporting matches, and other experimental fields. Chandler [22] launched a statistical study of the record values, record times and inter-record times. Applications of record values and their concomitants have been discussed in [23] and [24].

The record value is a special case of the $m$-GOSs with putting $m=-1$ and $k=1$. Therefore, the pdf and cdf for $R_{[r]}$ have been obtained as 


$$
\begin{aligned}
& g_{R_{[r]}}(y)=f_{Y}(y)\left[1+\alpha c_{r}\left(1-2 F_{Y}(y)\right)\right], \\
& G_{R_{[r]}}(y)=F_{Y}(y)\left[1+\alpha c_{r}\left(1-F_{Y}(y)\right)\right],
\end{aligned}
$$

where $c_{r}=2^{1-r}-1$ [See [23]]. Therefore, the extropy measure for $R_{[r]}$ is obtained as follows:

$$
J\left(R_{[r]}\right)=J(Y)-\frac{\alpha^{2} c_{r}^{2}}{2} \mathbb{E}\left[\frac{(1-2 U)^{2}}{q(U)}\right]-\alpha c_{r} \mathbb{E}\left[\frac{1-2 U}{q(U)}\right]
$$

Example 6 For the MTEWD, we can find

$$
J\left(R_{[r]}\right)=J(Y)\left[1-\alpha c_{r}\right]^{2}+\alpha^{2} c_{r}^{2} J(W)-\frac{16}{9}\left[\alpha^{2} c_{r}^{2}-\alpha c_{r}\right] J(V) .
$$

Example 7 For the MTED, we can find

$$
J\left(R_{[r]}\right)=\frac{1}{12 \theta_{2}}\left[3-\alpha^{2} c_{r}^{2}-2 \alpha c_{r}\right] .
$$

Also, we get

$$
A_{\alpha}(r)=J\left(R_{[r]}\right)-J\left(R_{[r-1]}\right)=\frac{\alpha\left(2^{-r}\right)}{\theta_{2}}\left[\alpha\left(2^{-r}-\frac{1}{3}\right)+\frac{1}{3}\right],
$$

which is positive, negative or zero whenever $0<\alpha \leq 1,-1 \leq \alpha<0$ or $\alpha=0$, respectively.

\subsection{CREX for $Y_{[r, n, m, k]}$}

For the concomitant $Y_{[r, n, m, k]}$ of the $r$ th $m$-GOS, the CREX measure is given by

$$
\begin{aligned}
\mathcal{J}^{\star}\left(Y_{[r, n, m, k]}\right)= & -\frac{1}{2} \int_{0}^{\infty} \bar{G}_{[r, n, m, k]}^{2}(y) d y \\
= & \mathcal{J}^{\star}(Y)-\frac{\left[\alpha C^{*}(r, n, m, k)\right]^{2}}{2} \mathbb{E}\left[q(U) U^{2}(1-U)^{2}\right] \\
& +\alpha C^{*}(r, n, m, k) \mathbb{E}\left[q(U) U(1-U)^{2}\right],
\end{aligned}
$$

where $\mathcal{J}^{\star}(Y)$ is the CREX of the random variable $Y$.

Case 1: If we put $m=0$ and $k=1$, then the CREX measure for $Y_{[r: n]}$ is presented as

$$
\mathcal{J}^{\star}\left(Y_{[r: n]}\right)=\mathcal{J}^{\star}(Y)-\frac{\left(\alpha \delta_{r}\right)^{2}}{2} \mathbb{E}\left[q(U)(U(1-U))^{2}\right]+\alpha \delta_{r} \mathbb{E}\left[q(U) U(1-U)^{2}\right] .
$$


Example 8 For the Morgenstern type bivariate uniform distribution (MTUD) with cdf

$$
F(x, y)=\frac{x y}{\theta_{1} \theta_{2}}\left[1+\alpha\left(1-\frac{x}{\theta_{1}}\right)\left(1-\frac{y}{\theta_{2}}\right)\right], \quad 0<x<\theta_{1}, \quad 0<y<\theta_{2},
$$

we have

$$
\mathcal{J}^{\star}\left(Y_{[r: n]}\right)=-\frac{\theta_{2}}{6}-\frac{\theta_{2}\left(\alpha \delta_{r}\right)^{2}}{60}+\frac{\theta_{2} \alpha \delta_{r}}{12}
$$

Therefore,

$$
D_{\alpha, \theta_{2}}(n)=\mathcal{J}^{\star}\left(Y_{[n: n]}\right)-\mathcal{J}^{\star}\left(Y_{[1: n]}\right)=\frac{-\alpha a_{n} \theta_{2}}{6},
$$

which is positive, negative or zero whenever $-1 \leq \alpha<0,0<\alpha \leq 1$ or $\alpha=0$, respectively.

Example 9 For the MTED, we have

$$
\mathcal{J}^{\star}\left(Y_{[r: n]}\right)=\frac{\theta_{2}}{12}\left[-3-2\left(\alpha \delta_{r}\right)^{2}+2 \alpha \delta_{r}\right] .
$$

Therefore,

$$
Q_{\alpha, \theta_{2}}(n)=\mathcal{J}^{\star}\left(Y_{[n: n]}\right)-\mathcal{J}^{\star}\left(Y_{[1: n]}\right)=\frac{-\alpha \theta_{2} a_{n}}{3},
$$

which is positive, negative or zero whenever $-1 \leq \alpha<0,0<\alpha \leq 1$ or $\alpha=0$, respectively.

Example 10 For the Morgenstern type bivariate Weibull distribution with the cdf

$$
\begin{aligned}
F(x, y)=\left(1-e^{-\theta_{1} x^{\beta_{1}}}\right)\left(1-e^{-\theta_{2} y^{\beta_{2}}}\right) & {\left[1+\alpha e^{-\theta_{1} x^{\beta_{1}}-\theta_{2} y^{\beta_{2}}}\right], } \\
x & >0, y>0, \beta_{i}>0, \theta_{i}>0, i=1,2,
\end{aligned}
$$

we have

$$
\begin{aligned}
\mathcal{J}^{\star}\left(Y_{[r: n]}\right)= & \left(\theta_{2}\right)^{\frac{-1}{\beta_{2}}}\left(\beta_{2}\right)^{-1} \Gamma\left(\frac{1}{\beta_{2}}\right) \\
& \times\left[-2^{\frac{-1}{\beta_{2}}-1}-\left(\alpha \delta_{r}\right)^{2}\left(2^{\frac{-1}{\beta_{2}}-1}+2^{\frac{-2}{\beta_{2}}-1}-3^{\frac{-1}{\beta_{2}}}\right)-\alpha \delta_{r}\left(3^{\frac{-1}{\beta_{2}}}-2^{\frac{-1}{\beta_{2}}}\right)\right] .
\end{aligned}
$$

Therefore,

$$
D_{\alpha, \theta_{2}}(n)=\mathcal{J}^{\star}\left(Y_{[n: n]}\right)-\mathcal{J}^{\star}\left(Y_{[1: n]}\right)=-2 \alpha a_{n}\left(\beta_{2}\right)^{-1} \Gamma\left(\frac{1}{\beta_{2}}\right)\left(\theta_{2}\right)^{\frac{-1}{\beta_{2}}}\left(-3^{\frac{-1}{\beta_{2}}}+2^{\frac{-1}{\beta_{2}}}\right),
$$


which is positive, negative or zero whenever $-1 \leq \alpha<0,0<\alpha \leq 1$ or $\alpha=0$, respectively.

Case 2: If we put $m=-1$ and $k=1$, then the CREX measure for $R_{[r]}$ is presented as

$$
\mathcal{J}^{\star}\left(R_{[r]}\right)=\mathcal{J}^{\star}(Y)-\frac{\alpha^{2} c_{r}^{2}}{2} \mathbb{E}\left[q(U)(U(1-U))^{2}\right]+\alpha c_{r} \mathbb{E}\left[q(U) U(1-U)^{2}\right] .
$$

\subsection{NCEX Measure for $Y_{[r, n, m, k]}$}

For the concomitant of the $r$ th $m$-GOS $Y_{[r, n, m, k]}$, the NCEX measure is given by

$$
\begin{aligned}
\mathcal{C} \mathcal{J}\left(Y_{[r, n, m, k]}\right)= & \mathcal{C} \mathcal{J}(Y)-\frac{\left[\alpha C^{*}(r, n, m, k)\right]^{2}}{2} \mathbb{E}\left[q(U)(U(1-U))^{2}\right] \\
& -\alpha C^{*}(r, n, m, k) \mathbb{E}\left[q(U) U^{2}(1-U)\right],
\end{aligned}
$$

where $\mathcal{C} \mathcal{J}(Y)$ is the NCEX of the random variable $Y$. It can be observed that the results are similar to CREX.

Case 1: If $m=0$ and $k=1$, then the NCEX measure for $Y_{[r: n]}$ in Morgenstern family is obtained as

$$
\mathcal{C} \mathcal{J}\left(Y_{[r: n]}\right)=\mathcal{C} \mathcal{J}(Y)-\frac{\left(\alpha \delta_{r}\right)^{2}}{2} \mathbb{E}\left[q(U)(U(1-U))^{2}\right]-\alpha \delta_{r} \mathbb{E}\left[U^{2}(1-U) q(U)\right] .
$$

Therefore, we have

$$
\begin{aligned}
& \mathcal{C J}\left(Y_{[n: n]}\right)=\mathcal{C} \mathcal{J}(Y)-\frac{\left(\alpha a_{n}\right)^{2}}{2} \mathbb{E}\left[q(U)(U(1-U))^{2}\right]+\alpha a_{n} \mathbb{E}\left[U^{2}(1-U) q(U)\right], \\
& \mathcal{C J}\left(Y_{[1: n]}\right)=\mathcal{C} \mathcal{J}(Y)-\frac{\left(\alpha a_{n}\right)^{2}}{2} \mathbb{E}\left[q(U)(U(1-U))^{2}\right]-\alpha a_{n} \mathbb{E}\left[U^{2}(1-U) q(U)\right] .
\end{aligned}
$$

Hence,

$$
\mathcal{C} \mathcal{J}\left(Y_{[n: n]}\right)-\mathcal{C} \mathcal{J}\left(Y_{[1: n]}\right)=2 \alpha a_{n} \mathbb{E}\left[U^{2}(1-U) q(U)\right]
$$

Case 2: If we put $m=-1$ and $k=1$, then the NCEX measure for $R_{[r]}$ is presented as

$$
\mathcal{C} \mathcal{J}\left(R_{[r]}\right)=\mathcal{C} \mathcal{J}(Y)-\frac{\alpha^{2} c_{r}^{2}}{2} \mathbb{E}\left[q(U)(U(1-U))^{2}\right]-\alpha c_{r} \mathbb{E}\left[q(U) U^{2}(1-U)\right] .
$$

\section{Empirical Measures for $Y_{[r, n, m, k]}$}

In this section, we estimate the NCREX and NCEX for concomitants by means of the empirical estimators. 


\subsection{Empirical NCREX}

Henceforward, we consider the problem of estimating the NCREX for concomitants using the empirical NCREX. Let $\left(X_{i}, Y_{i}\right), i=1,2, \cdots$, be a sequence from the Morgenstern family. According to (17) and relation $\mathcal{J}=-\mathcal{J}^{\star}$, the empirical NCREX of $Y_{[r, n, m, k]}$ can be obtained as follows:

$$
\begin{aligned}
\hat{J}\left(Y_{[r, n, m, k]}\right)= & \frac{1}{2} \int_{0}^{\infty}\left[1-\hat{G}_{[r, n, m, k]}(y)\right]^{2} d y \\
= & \frac{1}{2} \sum_{j=1}^{n-1} \int_{Z_{(j)}}^{Z_{(j+1)}}[1-\hat{F}(y)]^{2} d y \\
& +\frac{\left[\alpha C^{*}(r, n, m, k)\right]^{2}}{2} \sum_{j=1}^{n-1} \int_{Z_{(j)}}^{Z_{(j+1)}}[\hat{F}(y)(1-\hat{F}(y))]^{2} d y \\
& -\alpha C^{*}(r, n, m, k) \sum_{j=1}^{n-1} \int_{Z_{(j)}}^{Z_{(j+1)}} \hat{F}(y)[1-\hat{F}(y)]^{2} d y \\
= & \frac{1}{2} \sum_{j=1}^{n-1} U_{j}\left(1-\frac{j}{n}\right)^{2}+\frac{\left[\alpha C^{*}(r, n, m, k)\right]^{2}}{2} \sum_{j=1}^{n-1} U_{j}\left(\frac{j}{n}\left(1-\frac{j}{n}\right)\right)^{2} \\
& -\alpha C^{*}(r, n, m, k) \sum_{j=1}^{n-1} U_{j} \frac{j}{n}\left(1-\frac{j}{n}\right)^{2} \\
= & \frac{1}{2} \sum_{j=1}^{n-1} U_{j}\left(1-\frac{j}{n}\right)^{2}\left[1-\alpha C^{*}(r, n, m, k) \frac{j}{n}\right]^{2}
\end{aligned}
$$

where $U_{j}=Z_{(j+1)}-Z_{(j)}, j=1,2, \ldots, n-1$ are the sample spacings based on ordered random samples $Y_{j}$.

Case 1: If $m=0$ and $k=1$, then the empirical NCREX of $Y_{[r: n]}$ is given by

$$
\hat{\mathcal{J}}\left(Y_{[r: n]}\right)=\frac{1}{2} \sum_{j=1}^{n-1} U_{j}\left(1-\frac{j}{n}\right)^{2}\left[1-\alpha \delta_{r} \frac{j}{n}\right]^{2} .
$$

Case 2: If $m=-1$ and $k=1$, then the empirical NCREX of $R_{[r]}$ can be written as

$$
\hat{\mathcal{J}}\left(R_{[r]}\right)=\frac{1}{2} \sum_{j=1}^{n-1} U_{j}\left(1-\frac{j}{r}\right)^{2}\left[1-\alpha c_{r} \frac{j}{r}\right]^{2} .
$$

Let us consider the following examples.

Example 11 Let $\left(X_{i}, Y_{i}\right), i=1,2, \ldots, n$ be a random sample from MTED, then the sample spacings $U_{j}$ are independent and exponentially distributed with mean $\frac{1}{\theta_{2}(n-j)}$ (for more details see [25]). Upon recalling (22), we obtain 


$$
\begin{aligned}
E\left[\hat{\mathcal{J}}\left(Y_{[r: n]}\right)\right] & =\frac{1}{2 \theta_{2}} \sum_{j=1}^{n-1} \frac{n-j}{n^{2}}\left[1-\alpha \delta_{r} \frac{j}{n}\right]^{2}, \\
\operatorname{Var}\left[\hat{\mathcal{J}}\left(Y_{[r: n]}\right)\right] & =\frac{1}{4 n^{4} \theta_{2}^{2}} \sum_{j=1}^{n-1}(n-j)^{2}\left[1-\alpha \delta_{r} \frac{j}{n}\right]^{4},
\end{aligned}
$$

respectively. We computed the values of $E\left[\hat{\mathcal{J}}\left(Y_{[2: n]}\right)\right]$ and $\operatorname{Var}\left[\hat{\mathcal{J}}\left(Y_{[2: n]}\right)\right]$ for sample sizes $n=5,10,15,20, \theta_{2}=0.5,1,2, \alpha=-1,-0.5,0.5,1$ in Table 1 . We can easily see that $E\left[\hat{\mathcal{J}}\left(Y_{[2: n]}\right)\right]$ and $\operatorname{Var}\left[\hat{\mathcal{J}}\left(Y_{[2: n]}\right)\right]$ are decreasing in $\alpha$. Also, $\lim _{n \rightarrow \infty} \operatorname{Var}\left[\hat{\mathcal{J}}\left(Y_{[2: n]}\right)\right]=0$.

Example 12 For MTUD with $\theta_{1}=\theta_{2}=1$, the sample spacings $U_{j}$ are independent of beta distribution with parameters 1 and $n$ [for more details see [25]]. By making use of (22), we obtain

$$
\begin{gathered}
E\left[\hat{\mathcal{J}}\left(R_{[r]}\right)\right]=\frac{1}{n+1} \sum_{j=1}^{n-1}\left(1-\frac{j}{n}\right)^{2}\left[1-\alpha c_{r} \frac{j}{n}\right]^{2}, \\
\operatorname{Var}\left[\hat{\mathcal{J}}\left(R_{[r]}\right)\right]=\frac{n}{(n+1)^{2}(n+2)} \sum_{j=1}^{n-1}\left(1-\frac{j}{n}\right)^{4}\left[1-\alpha c_{r} \frac{j}{n}\right]^{4} .
\end{gathered}
$$

We computed the values of $E\left[\hat{\mathcal{J}}\left(R_{[2]}\right)\right]$ and $\operatorname{Var}\left[\hat{\mathcal{J}}\left(R_{[2]}\right)\right]$ for sample sizes $n=5,10,15,20, \alpha=-1,-0.5,0.5,1$ in Table 2 . We can easily see that $E\left[\hat{\mathcal{J}}\left(R_{[2]}\right)\right]$ and $\operatorname{Var}\left[\hat{\mathcal{J}}\left(R_{[2]}\right)\right]$ are decreasing in $\alpha$. Also, $\lim _{n \rightarrow \infty} \operatorname{Var}\left[\hat{\mathcal{J}}\left(R_{[2]}\right)\right]=0$.

Theorem 1 Let $\left(X_{i}, Y_{i}\right), i=1,2, \cdots, n$ be a random sample of size $n$ from Morgenstern family. Then

$$
\hat{\mathcal{J}}\left(R_{[r]}\right) \longrightarrow \mathcal{J}\left(R_{[r]}\right) \text { as } n \rightarrow \infty
$$

We are now capable to present a central limit theorem for $\hat{\mathcal{J}}\left(R_{[r]}\right)$ coming from a random sample of the MTED.

Theorem 2 Let $\left(X_{i}, Y_{i}\right), i=1,2, \ldots, n$ be a random sample from MTED. Then

$$
Z_{n}=\frac{\hat{\mathcal{J}}\left(R_{[r]}\right)-E\left[\hat{\mathcal{J}}\left(R_{[r]}\right)\right]}{\sqrt{\operatorname{Var}\left[\hat{\mathcal{J}}\left(R_{[r]}\right)\right]}}
$$

converges in distribution to a standard normal distribution. 
Table 1 Numerical values of $\mathbb{E}\left[\hat{\mathcal{J}}\left(Y_{[2: n]}\right)\right]$ and $\operatorname{Var}\left[\hat{\mathcal{J}}\left(Y_{[2: n]}\right)\right]$ for MTED

\begin{tabular}{|c|c|c|c|c|c|c|}
\hline \multirow[b]{2}{*}{$\theta_{2}$} & \multicolumn{6}{|c|}{$\mathbb{E}\left[\hat{\mathcal{J}}\left(Y_{[2: n]}\right)\right]$} \\
\hline & 0.5 & 1 & 2 & 0.5 & 1 & 2 \\
\hline$n$ & $\alpha=-1$ & & & $\alpha=-0.5$ & & \\
\hline 5 & 0.515 & 0.257 & 0.128 & 0.455 & 0.227 & 0.113 \\
\hline 10 & 0.693 & 0.346 & 0.173 & 0.653 & 0.281 & 0.140 \\
\hline 15 & 0.762 & 0.381 & 0.190 & 0.602 & 0.301 & 0.150 \\
\hline \multirow[t]{2}{*}{20} & 0.798 & 0.399 & 0.199 & 0.623 & 0.311 & 0.155 \\
\hline & \multicolumn{3}{|l|}{$\alpha=0.5$} & \multicolumn{3}{|l|}{$\alpha=1$} \\
\hline 5 & 0.348 & 0.174 & 0.872 & 0.302 & 0.151 & 0.075 \\
\hline 10 & 0.353 & 0.1766 & 0.0883 & 0.273 & 0.136 & 0.068 \\
\hline 15 & 0.3538 & 0.1769 & 0.0884 & 0.264 & 0.132 & 0.066 \\
\hline \multirow[t]{3}{*}{20} & 0.354 & 0.177 & 0.0885 & 0.260 & 0.130 & 0.065 \\
\hline & \multicolumn{6}{|c|}{$\operatorname{Var}\left[\hat{\mathcal{J}}\left(Y_{[2: n]}\right)\right]$} \\
\hline & $\alpha=-1$ & & & $\alpha=-0.5$ & & \\
\hline 5 & 0.074 & 0.018 & 0.004 & 0.059 & 0.014 & 0.003 \\
\hline 10 & 0.059 & 0.014 & 0.003 & 0.041 & 0.010 & 0.002 \\
\hline 15 & 0.046 & 0.011 & 0.0028 & 0.030 & 0.007 & 0.0019 \\
\hline \multirow[t]{2}{*}{20} & 0.037 & 0.009 & 0.0023 & 0.024 & 0.006 & 0.0015 \\
\hline & \multicolumn{3}{|l|}{$\alpha=0.5$} & \multicolumn{3}{|l|}{$\alpha=1$} \\
\hline 5 & 0.038 & 0.009 & 0.002 & 0.030 & 0.007 & 0.0019 \\
\hline 10 & 0.019 & 0.004 & 0.001 & 0.014 & 0.003 & 0.0008 \\
\hline 15 & 0.013 & 0.003 & 0.0008 & 0.009 & 0.002 & 0.0005 \\
\hline 20 & 0.010 & 0.002 & 0.0006 & 0.006 & 0.0017 & 0.0004 \\
\hline
\end{tabular}

Table 2 Numerical values of $\mathbb{E}\left[\hat{\mathcal{J}}\left(R_{[2]}\right)\right]$ and $\operatorname{Var}\left[\hat{\mathcal{J}}\left(R_{[2]}\right)\right]$ for MTUD with $\theta_{1}=\theta_{2}=1$

\begin{tabular}{|c|c|c|c|c|c|c|c|c|}
\hline \multirow[t]{2}{*}{$n$} & \multicolumn{4}{|c|}{$\mathbb{E}\left[\hat{\mathcal{J}}\left(R_{[2]}\right)\right]$} & \multicolumn{4}{|c|}{$\operatorname{Var}\left[\hat{\mathcal{J}}\left(R_{[2]}\right)\right]$} \\
\hline & $\alpha=-1$ & $\alpha=-0.5$ & $\alpha=0.5$ & $\alpha=1$ & $\alpha=-1$ & $\alpha=-0.5$ & $\alpha=0.5$ & $\alpha=1$ \\
\hline 5 & 0.233 & 0.216 & 0.183 & 0.166 & 0.014 & 0.013 & 0.010 & 0.008 \\
\hline 10 & 0.296 & 0.277 & 0.240 & 0.221 & 0.012 & 0.011 & 0.009 & 0.008 \\
\hline 15 & 0.320 & 0.301 & 0.262 & 0.243 & 0.010 & 0.009 & 0.008 & 0.007 \\
\hline 20 & 0.333 & 0.313 & 0.274 & 0.254 & 0.008 & 0.007 & 0.006 & 0.005 \\
\hline
\end{tabular}

Proof The mean of empirical NCREX measure $\hat{\mathcal{J}}\left(R_{[r]}\right)$ can be expressed as sum of independent random variables as 


$$
\hat{\mathcal{J}}\left(R_{[r]}\right)=\sum_{j=1}^{n-1} W_{j}
$$

where $W_{j}=\frac{1}{2} U_{j}\left(1-\frac{j}{n}\right)^{2}\left[1-\alpha c_{r} \frac{j}{n}\right]^{2}$ are independent random variables with the mean and variance given by

$$
\begin{gathered}
E\left[W_{j}\right]=\frac{1}{2 n \theta_{2}}\left(1-\frac{j}{n}\right)\left[1-\alpha c_{r} \frac{j}{n}\right]^{2}, \\
\operatorname{Var}\left[W_{j}\right]=\frac{1}{4 n^{2} \theta_{2}^{2}}\left(1-\frac{j}{n}\right)^{2}\left[1-\alpha c_{r} \frac{j}{n}\right]^{4} .
\end{gathered}
$$

Since $E\left[\left|W_{j}-E\left(W_{j}\right)\right|^{3}\right]=2 e^{-1}(6-e)\left[E\left(W_{j}\right)\right]^{3}$ for any exponentially distributed random variable $W_{j}$, by setting $\alpha_{j, k}=E\left[\left|W_{j}-E\left(W_{j}\right)\right|^{k}\right]$ the following approximations hold for large $n$ :

$$
\begin{aligned}
& \sum_{j=1}^{n} \alpha_{j, 2}=\frac{1}{4 n^{2} \theta_{2}^{2}} \sum_{j=1}^{n}\left(1-\frac{j}{n}\right)^{2}\left[1-\alpha c_{r} \frac{j}{n}\right]^{4} \approx \frac{c_{2}}{4 n \theta_{2}^{2}}, \\
& \sum_{j=1}^{n} \alpha_{j, 3}=\frac{2(6-e)}{e n^{3} \theta_{2}^{3}} \sum_{j=1}^{n}\left(1-\frac{j}{n}\right)^{3}\left[1-\alpha c_{r} \frac{j}{n}\right]^{6} \approx \frac{2(6-e) c_{3}}{e n^{2} \theta_{2}^{3}},
\end{aligned}
$$

where $c_{k}:=\int_{0}^{1}(1-x)^{k}\left[1-\alpha c_{r} x\right]^{2 k}$. Hence, Lyapunov's condition of the central limit theorem is satisfied [cf. [26]]:

$$
\frac{\left(\alpha_{1,3}+\cdots+\alpha_{n, 3}\right)^{1 / 3}}{\left(\alpha_{1,2}+\cdots+\alpha_{n, 2}\right)^{1 / 2}} \approx \frac{\left[2(6-e) c_{3}\right]^{1 / 3}}{e^{1 / 3} c_{2}^{1 / 2}} n^{-1 / 6} \rightarrow 0 \quad \text { as } n \rightarrow \infty,
$$

which completes the proof.

If the random variables $\left(X_{i}, Y_{i}\right), i=1,2, \cdots, n$ are from MTED. Then, upon using the results of Theorem 3.2, an approximate confidence interval for $\mathcal{J}\left(R_{[r]}\right)$ can be constructed as

$$
\hat{\mathcal{J}}\left(R_{[r]}\right) \pm z_{q / 2} \sqrt{\operatorname{Var}\left[\hat{\mathcal{J}}\left(R_{[r]}\right)\right]}
$$

where $z_{q}$ is the $q$ th upper quantile of the standard normal distribution.

\subsection{Empirical NCEX for Concomitants of $m$-GOSs}

In this section, we address the problem of estimating the NCEX for concomitants using the empirical NCEX. Let $\left(X_{i}, Y_{i}\right), i=1,2, \cdots, n$ be a random sample of size $n$ from the Morgenstern family. According to (19), the empirical NCEX of $Y_{[r, n, m, k]}$ can be obtained as 


$$
\begin{aligned}
\widehat{\mathcal{C J}}\left(Y_{[r, n, m, k]}\right)= & \frac{1}{2} \sum_{j=1}^{n-1} U_{j}\left(1-\left(\frac{j}{n}\right)^{2}\right) \\
& -\frac{\left[\alpha C^{*}(r, n, m, k)\right]^{2}}{2} \sum_{j=1}^{n-1} U_{j}\left(\frac{j}{n}\left(1-\frac{j}{n}\right)\right)^{2} \\
& -\alpha C^{*}(r, n, m, k) \sum_{j=1}^{n-1} U_{j}\left(\frac{j}{n}\right)^{2}\left(1-\frac{j}{n}\right) .
\end{aligned}
$$

Case 1: If $m=0$ and $k=1$, then the empirical NCEX of $Y_{[r: n]}$ is given by

$$
\widehat{\mathcal{C J}}\left(Y_{[r: n]}\right)=\frac{1}{2} \sum_{j=1}^{n-1} U_{j}\left[1-\left(\frac{j}{n}\right)^{2}\left[1+\alpha \delta_{r}\left(1-\frac{j}{n}\right)\right]^{2}\right] .
$$

Case 2: If $m=-1$ and $k=1$, then the empirical NCEX of $R_{[r]}$ can be written as

$$
\widehat{\mathcal{C J}}\left(R_{[r]}\right)=\frac{1}{2} \sum_{j=1}^{n-1} U_{j}\left[1-\left(\frac{j}{n}\right)^{2}\left[1+\alpha c_{r}\left(1-\frac{j}{n}\right)\right]^{2}\right] .
$$

Example 13 For MTED, from (27), we obtain

$$
\begin{gathered}
E\left[\widehat{\mathcal{C J}}\left(R_{[r]}\right)\right]=\frac{1}{2 \theta_{2}} \sum_{j=1}^{n-1} \frac{1}{(n-j)}\left[1-\left(\frac{j}{n}\right)^{2}\left[1+\alpha c_{r}\left(1-\frac{j}{n}\right)\right]^{2}\right], \\
\operatorname{Var}\left[\widehat{\mathcal{C J}}\left(R_{[r]}\right)\right]=\frac{1}{4 \theta_{2}^{2}} \sum_{j=1}^{n-1}\left(\frac{1}{(n-j)}\right)^{2}\left[1-\left(\frac{j}{n}\right)^{2}\left[1+\alpha c_{r}\left(1-\frac{j}{n}\right)\right]^{2}\right]^{2} .
\end{gathered}
$$

We computed the values of $E\left[\widehat{\mathcal{C J}}\left(R_{[2]}\right)\right]$ and $\operatorname{Var}\left[\widehat{\mathcal{C J}}\left(R_{[2]}\right)\right]$ for sample sizes $n=5,10,15,20, \theta_{2}=0.5,1,2, \alpha=-1,-0.5,0.5,1$ in Table 3 . We can easily see that $E\left[\widehat{\mathcal{C J}}\left(R_{[2]}\right)\right]$ and $\operatorname{Var}\left[\widehat{\mathcal{C J}}\left(R_{[2]}\right)\right]$ are decreasing in $\alpha$. Also, $\lim _{n \rightarrow \infty} \operatorname{Var}\left[\widehat{\mathcal{C J}}\left(R_{[2]}\right)\right]=0$.

\section{Real Data}

We consider a data set on 137 bone marrow transplant (BMT) patients presented by [27]. It is included 22 attributes. The attribute ' $T_{2}$ ' represents disease free survival time (time to relapse, death or end of study), and the attribute ' $T_{P}$ ' represents time (in days) to return of platelets to normal levels. For $T_{2}$ and $T_{P}$, the Spearman correlation coefficient is -0.2544 (with p-value 0.0027 ), and the Kendall correlation coefficient is -0.1806 (with p-value 0.0020 ). [28] analyzed these two attributes and fitted some families of bivariate exponential distributions. For the MTED with cdf in (14), the maximum likelihood estimation of parameters 
Table 3 Numerical values of $\mathbb{E}\left[\widehat{\mathcal{C J}}\left(R_{[2]}\right)\right]$ and $\operatorname{Var}\left[\widehat{\mathcal{C J}}\left(R_{[2]}\right)\right]$ for MTED

\begin{tabular}{|c|c|c|c|c|c|c|}
\hline \multirow{3}{*}{$\begin{array}{l}\theta_{2} \\
n\end{array}$} & \multicolumn{6}{|c|}{$\mathbb{E}\left[\widehat{\mathcal{C J}}\left(R_{[2]}\right)\right]$} \\
\hline & 0.5 & 1 & 2 & 0.5 & 1 & 2 \\
\hline & \multicolumn{3}{|l|}{$\alpha=-1$} & \multicolumn{3}{|c|}{$\alpha=-0.5$} \\
\hline 5 & 1.420 & 0.710 & 0.355 & 1.315 & 0.657 & 0.328 \\
\hline 10 & 1.614 & 0.807 & 0.4035 & 1.487 & 0.743 & 0.371 \\
\hline 15 & 1.680 & 0.840 & 0.420 & 1.545 & 0.772 & 0.386 \\
\hline \multirow[t]{2}{*}{20} & 1.712 & 0.856 & 0.428 & 1.574 & 0.787 & 0.393 \\
\hline & \multicolumn{3}{|l|}{$\alpha=0.5$} & \multicolumn{3}{|l|}{$\alpha=1$} \\
\hline 5 & 1.075 & 0.537 & 0.268 & 0.940 & 0.470 & 0.235 \\
\hline 10 & 1.202 & 0.601 & 0.300 & 1.044 & 0.522 & 0.261 \\
\hline 15 & 1.244 & 0.622 & 0.311 & 1.078 & 0.539 & 0.269 \\
\hline \multirow[t]{3}{*}{20} & 1.265 & 0.632 & 0.316 & 1.095 & 0.547 & 0.273 \\
\hline & \multicolumn{6}{|c|}{$\underline{\operatorname{Var}\left[\widehat{\mathcal{C J}}\left(R_{[2]}\right)\right]}$} \\
\hline & \multicolumn{3}{|c|}{$\alpha=-1$} & \multicolumn{3}{|c|}{$\alpha=-0.5$} \\
\hline 5 & 0.535 & 0.133 & 0.033 & 0.450 & 0.112 & 0.028 \\
\hline 10 & 0.313 & 0.078 & 0.019 & 0.259 & 0.064 & 0.016 \\
\hline 15 & 0.219 & 0.054 & 0.013 & 0.180 & 0.045 & 0.011 \\
\hline \multirow[t]{2}{*}{20} & 0.168 & 0.042 & 0.010 & 0.138 & 0.034 & 0.008 \\
\hline & \multicolumn{3}{|l|}{$\alpha=0.5$} & \multicolumn{3}{|l|}{$\alpha=1$} \\
\hline 5 & 0.290 & 0.072 & 0.018 & 0.221 & 0.055 & 0.013 \\
\hline 10 & 0.162 & 0.040 & 0.010 & 0.121 & 0.030 & 0.007 \\
\hline 15 & 0.11 & 0.027 & 0.006 & 0.083 & 0.020 & 0.005 \\
\hline 20 & 0.085 & 0.021 & 0.005 & 0.063 & 0.015 & 0.003 \\
\hline
\end{tabular}

are $\hat{\alpha}=-0.6703, \hat{\theta}_{1}=47.9682$, and $\hat{\theta}_{2}=730.8846$. We calculated the NCREX of $Y_{[r, n]}$ for MTED given in (18) and the empirical NCREX of $Y_{[r, n]}$ given in (21) for all values of $r$ and some values of $\alpha$. The results are given in Fig. 1. We can observe that

1. The values of NCREX and empirical NCREX are close when $-0.5 \leq \alpha \leq 0.5$ especially when $\alpha=0$ for all values of $r$.

2. When $\alpha>0.5$, the values of NCREX and empirical NCREX are close for $r \geq 50$

3. When $\alpha>0.5$, the value of NCREX is larger than the value of empirical NCREX for $r<50$.

4. When $\alpha<-0.5$, the values of NCREX and empirical NCREX are close for $r \leq 100$. 

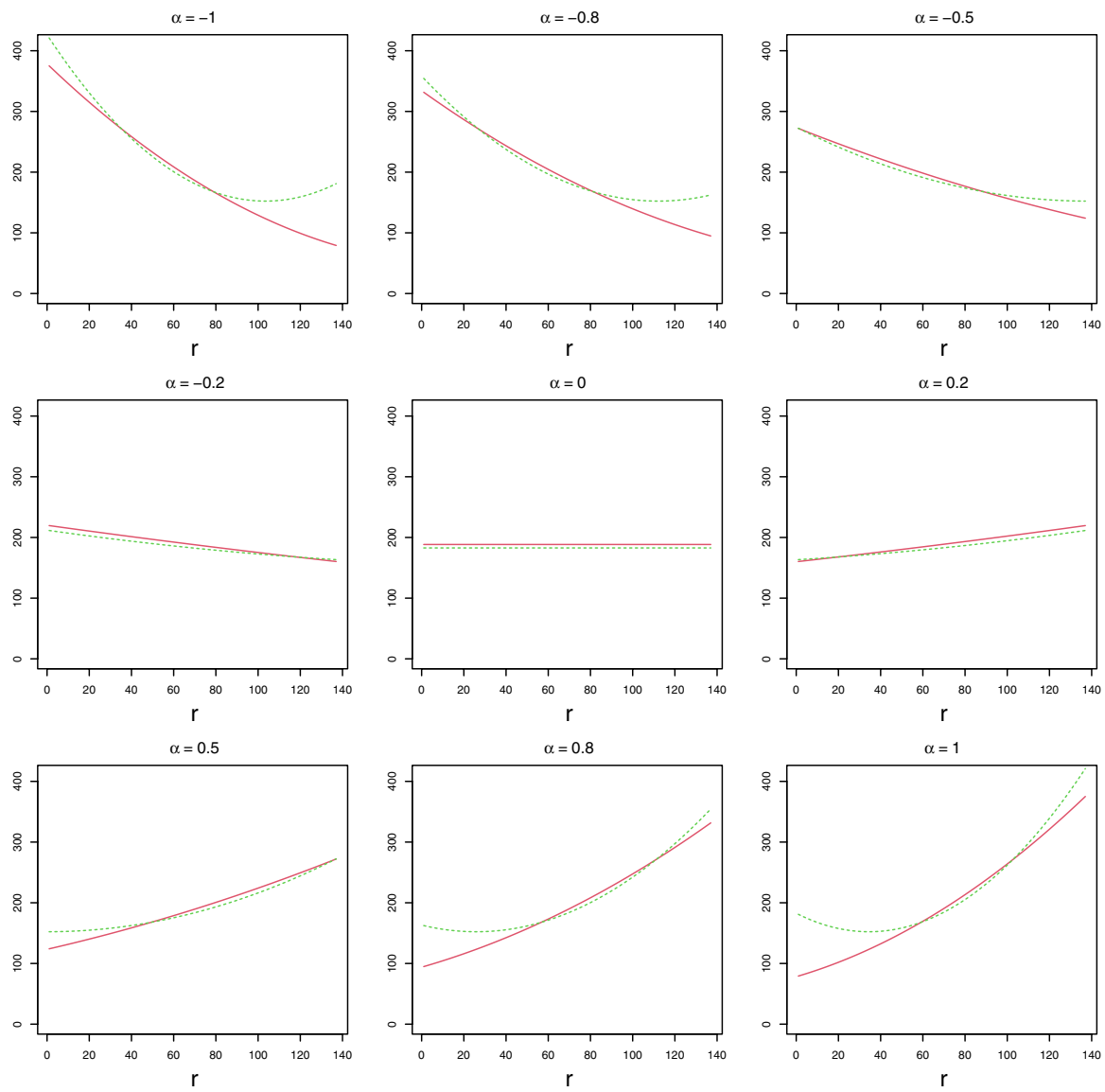

Fig. 1 The NCREX for MTED of $Y_{[r, n]}$ (dashed line) and empirical NCREX (solid line) of $Y_{[r, n]}$

5. When $\alpha<-0.5$, the value of NCREX is larger than the value of empirical NCREX for $r>100$.

\section{Conclusions}

In this paper, we have obtained various properties of extropy measure for concomitants of $m$-GOSs in Morgenstern family. Applications of this result have been given for concomitants of order statistics and record values. Also, we have introduced CREX and NCEX for $G_{[r ; n ; m ; k]}(y)$ in this family. These measures can be applied for measuring the information volatility contained in concomitants of order statistics and record values. Finally, we have proposed the estimators of NCREX and NCEX for concomitants by using empirical approach. Further, the validity of the new 
measures have supported by numerical simulations on MTED and MTUD. Using a real data set, we showed that the NCREX of $Y_{[r, n]}$ for MTED and the empirical NCREX of $Y_{[r, n]}$ are close in most cases.

Acknowledgements The authors are grateful to the anonymous referees for the helpful comments and suggestions to improve this manuscript.

Open Access This article is distributed under the terms of the Creative Commons Attribution 4.0 International License (http://creativecommons.org/licenses/by/4.0/), which permits unrestricted use, distribution, and reproduction in any medium, provided you give appropriate credit to the original author(s) and the source, provide a link to the Creative Commons license, and indicate if changes were made.

Author Contributions The authors have equally made contributions. All authors read and approved the final manuscript.

Funding The authors state that no funding source for this paper.

Availability of Data and Materials Not applicable.

\section{Declarations}

Conflict of interest The authors declare that they have no competing interests. The authors state that no funding source or sponsor has participated in the realization of this work.

Open Access This article is licensed under a Creative Commons Attribution 4.0 International License, which permits use, sharing, adaptation, distribution and reproduction in any medium or format, as long as you give appropriate credit to the original author(s) and the source, provide a link to the Creative Commons licence, and indicate if changes were made. The images or other third party material in this article are included in the article's Creative Commons licence, unless indicated otherwise in a credit line to the material. If material is not included in the article's Creative Commons licence and your intended use is not permitted by statutory regulation or exceeds the permitted use, you will need to obtain permission directly from the copyright holder. To view a copy of this licence, visit http://creativecommons.org/ licenses/by/4.0/.

\section{References}

1. Kamps, U.: A concept of generalized order statistics. J. Stat. Plann. Inference 48(1), 1-23 (1995)

2. Morgenstern, D.: Einfache beispiele zweidimensionaler verteilungen. Mitteilingsblatt für Mathematishe Statistik 8(1), 234-235 (1956)

3. Beg, M.I., Ahsanullah, M.: Concomitants of generalized order statistics from Farlie-Gumbel-Morgenstern distributions. Stat. Methodol. 5(1), 1-20 (2008)

4. Tahmasebi, S., Jafari, A.A., Ahsanullah, M.: Properties on concomitants of generalized order statistics from a bivariate Rayleigh distribution. Bull. Malays. Math. Sci. Soc. 41(1), 355-370 (2018)

5. Daneshi, S., Nezakati, A., Tahmasebi, S., Longobardi, M.: Inaccuracy measures for concomitants of generalized order statistics in Morgenstern family. Note di Matematica 40(2), 83-98 (2021)

6. Barakat, H.M., Husseiny, I.A.: Some information measures in concomitants of generalized order statistics under iterated Farlie-Gumbel-Morgenstern bivariate type. Quaestiones Mathematicae 44(5), 581-598 (2021)

7. Abd Elgawad, M.A., Alawady, M.A., Barakat, H.M., Xiong, S.: Concomitants of generalized order statistics from Huang-Kotz Farlie-Gumbel-Morgenstern bivariate distribution: Some information measures. Bull. Malays. Math. Sci. Soc. 43, 2627-2645 (2020) 
8. Abd Elgawad, M.A., Barakat, H.M., Alawady, M.A.: Concomitants of generalized order statistics from bivariate Cambanis family: Some information measures. Bull. Iran. Math. Soc. (2021). https:// doi.org/10.1007/s41980-021-00532-8

9. Alawady, M. A., Barakat, H. M., and Abd Elgawad, M. A.: Concomitants of generalized order statistics from bivariate Cambanis family of distributions under a general setting. Bull. Malays. Math. Sci. Soc., 44:3129-3159 (2021)

10. Mohamed, M.S., Abdulrahman, A.T., Almaspoor, Z., Yusuf, M.: Ordered variables and their concomitants under extropy via COVID-19 data application. Complexity (2021). https://doi.org/10. $1155 / 2021 / 6491817$

11. Lad, F., Sanfilippo, G., Agro, G.: Extropy: Complementary dual of entropy. Stat. Sci. 30(1), 40-58 (2015)

12. Qiu, G.: The extropy of order statistics and record values. Stat. Probab. Lett. 120, 52-60 (2017)

13. Qiu, G., Jia, K.: The residual extropy of order statistics. Stat. Probab. Lett. 133, 15-22 (2018)

14. Qiu, G., Jia, K.: Extropy estimators with applications in testing uniformity. J. Nonparametric Stat. 30(1), 182-196 (2018)

15. Qiu, G., Wang, L., Wang, X.: On extropy properties of mixed systems. Probab. Eng. Inf. Sci. 33(3), 471-486 (2019)

16. Zamanzade, E. and Mahdizadeh, M.: Extropy estimation in ranked set sampling with its application in testing uniformity. In Ranked Set Sampling, pages 259-267. Elsevier (2019)

17. Mohamed, M.S., Barakat, H.M., Alyami, S.A., AbdElgawad, M.A.: Fractional entropy-based test of uniformity with power comparisons. J. Math. (2021). https://doi.org/10.1155/2021/5331260

18. Jahanshahi, S., Zarei, H., Khammar, A.: On cumulative residual extropy. Probab. Eng. Inf. Sci. 34(4), 605-625 (2020)

19. Abdul Sathar, E. I. and Nair, D.: On dynamic survival extropy. Commun. Stat.-Theory Methods, 50(6), 1295-1313 (2020)

20. Tahmasebi, S., Toomaj, A.: On negative cumulative extropy with applications. Commun. Stat. Theory Methods (2021). https://doi.org/10.1080/03610926.2020.1831541

21. Eryilmaz, S.: Concomitants in a sequence of independent nonidentically distributed random vectors. Commun. Stat. Theory Methods 34(9-10), 1925-1933 (2005)

22. Chandler, K.N.: The distribution and frequency of record values. J. R. Stat. Soc. Ser. B (Methodol) 14, 220-228 (1952)

23. Houchens, R.L.: Record Value Theory and Inference. ProQuest LLC, Ann Arbor (1984)

24. Arnold, B., Balakrishnan, N., Nagaraja, H.: Records. John Wiley \& Sons Inc, New York (1998)

25. Pyke, R.: Spacings. J. Roy. Stat. Soc.: Ser. B (Methodol.) 27(3), 395-436 (1965)

26. Billingsley, P.: Probability and Measure. John Wiley \& Sons. (2008)

27. Klein, J.P., Moeschberger, M.L.: Survival Analysis, 2nd edn. Springer, New York (2003)

28. Sharifonnasabi, Z., Alamatsaz, M.H., Kazemi, I.: On Properties of a class of bivariate FGM type distributions. J. Stat. Res. Iran 15(2), 275-300 (2019) 